\title{
A Thermodynamic Method of Intuitionistic Fuzzy MCDM to Assist the Hierarchical Medical System in China
}

DOI:

10.1016/j.ins.2017.08.070

\section{Document Version}

Accepted author manuscript

Link to publication record in Manchester Research Explorer

\section{Citation for published version (APA):}

Ren, P., Liao, H., \& Zeng, X. (2017). A Thermodynamic Method of Intuitionistic Fuzzy MCDM to Assist the Hierarchical Medical System in China. Information Sciences. https://doi.org/10.1016/j.ins.2017.08.070

\section{Published in:}

Information Sciences

\section{Citing this paper}

Please note that where the full-text provided on Manchester Research Explorer is the Author Accepted Manuscript or Proof version this may differ from the final Published version. If citing, it is advised that you check and use the publisher's definitive version.

\section{General rights}

Copyright and moral rights for the publications made accessible in the Research Explorer are retained by the authors and/or other copyright owners and it is a condition of accessing publications that users recognise and abide by the legal requirements associated with these rights.

\section{Takedown policy}

If you believe that this document breaches copyright please refer to the University of Manchester's Takedown Procedures [http://man.ac.uk/04Y6Bo] or contact uml.scholarlycommunications@manchester.ac.uk providing relevant details, so we can investigate your claim.

\section{OPEN ACCESS}




\title{
A Thermodynamic Method of Intuitionistic Fuzzy MCDM to Assist the Hierarchical Medical System in China
}

\author{
Peijia Ren ${ }^{\mathrm{a}, \mathrm{b}}$, Zeshui Xu ${ }^{\mathrm{a}, *}$, Huchang Liao ${ }^{\mathrm{a}}$, Xiao-Jun Zeng ${ }^{\mathrm{b}}$ \\ ${ }^{a}$ Business School, Sichuan University, Chengdu, Sichuan 610064, China \\ ${ }^{b}$ School of Computer Science, University of Manchester, M13 9PL, U.K.
}

\begin{abstract}
Thermodynamics is of knowledge structure to depict the stability (or instability) of a system effectively. For the increasing complexity of the application problems and the fuzzy nature of human thought, intuitionistic fuzzy sets were proposed and have become a powerful tool to portray the uncertainty of things. In order to best utilize decision information to solve the multi-criteria decision making (MCDM) problems in an intuitionistic fuzzy environment, the paper proposes a thermodynamic method for MCDM with intuitionistic fuzzy numbers (IFNs), which not only utilizes the quantity of the decision information to make decision, but also concerns the quality of the decision information in the decision making process. Then, we conduct simulations to compare the decision making results derived by the proposed method and other two commonly used decision making methods - Weighted averaging operator (WAO) and TOPSIS in the intuitionistic fuzzy environment. Further, the thermodynamic method for MCDM with IFNs is applied to assist the hierarchical medical system in West China Hospital as a case study.
\end{abstract}

\footnotetext{
* Corresponding author. E-mail addresses: renpeijia@outlook.com, xuzeshui@263.net, liaohuchang@163.com, x.zeng@manchester.ac.uk.
} 
The empirical results derived from the simulations and applications show: (1) If the optimal selections obtained from the proposed method and one of the two commonly used methods are same, then it is likely that the three decision making methods would obtain same results; (2) If the optimal selections by the three method are different, then it is often the cases where the selection by the proposed method is different from the two other methods whereas the selections by the two other methods are the same. The reason behind is the unique feature of the proposed method in which the quality of the decision making information is taken into consideration. These empirical studies demonstrate that the quality of the decision making information has significant impacts on the decision making results, which indicates that the proposed method is effective to be utilized in practical decision making problems.

Keywords: Multi-criteria decision making; Intuitionistic fuzzy set; Thermodynamics; Information quantity; Information quality; Weighted averaging operator, TOPSIS, Hierarchical medical system.

\section{Introduction}

Multi-criteria decision making (MCDM) usually refers to the process of ranking the alternatives or selecting a desirable alternative among a collection of possible alternatives with respect to a list of criteria. Under the pluralistic and complicated social environment, the theories of MCDM have been rapidly developed and widely applied in various fields of engineering project [1-4], military affair [5-7], management [8-11] and so on. Considering the large application foreground of MCDM, many decision making methods have been proposed to deal with the MCDM problems, like 
TOPSIS (Technique for Order Preference by Similarity to an Ideal Solution) [12], ELECTRE (Elimination and Choice Expressing REality) [13], VIKOR (vlsekriterijumska optimizacija i kompromisno resenje in serbian, meaning multi-criteria optimization and compromise solution) [14], TODIM (an acronym in Portuguese for Interactive Multi-criteria Decision Making) [15], COPRAS (Complex Proportional Assessment) [16], etc.

The above methods generally transform the original decision information into the forms that can be compared visually. Even though they manage to rank the alternatives via a proper decision making procedure, it only catches one character of the decision information, i.e., the numerical values. However, in some cases, the numerical values are not adequate to represent the decision information. For example, if four decision makers (DMs) with same weights provide the decision information to object 1 as $0.2,0.6,0.7,0.1$, and give the decision information to object 2 as $0.4,0.2$, $0.6,0.4$, then by the weighted arithmetic mean [17], the final scores of these two objects are both 0.4. Obviously, the two sets of assessments are different, and the decision information of object 2 is more dependable since its distribution is more concentrated. Therefore, the distribution of the decision information, as a significant character, should be taken into consideration in the decision making process.

Thermodynamics, a branch of physics, has effective knowledge structure to depict the stability (or instability) of a system. More specifically, the physical parameters like energy [18], exergy [19], entropy [20] and the three laws of thermodynamics [21-23], can reflect the quantity and the quality of a system from the viewpoint of thermodynamics. To date, the majority of the decision making methods just consider the quantity of the decision values, but ignore the distribution of them. Due to the complexity of applications and the limitations of human thought, it is often very hard for DMs 
to collect much information for a decision making problem or it may cost much resource and time to obtain more information for the problem. Therefore, it is very crucial to make the best use of the available information in practice. Fortunately, the knowledge of thermodynamics processes the capability to take into account the quantity and quality of information, which is very interesting and fruitful to be utilized in decision making process. Toward this end, we endeavor to extend the knowledge of thermodynamics to handle decision making problems, which contains analogizing decision information firstly according to the thermodynamic parameters, and then extending the thought of thermodynamics to the decision making process so as to obtain a reliable and high quality decision making result.

With the increasing diversity and complexity of the application problems in this day and age, there always exist some uncertainties for the objects. Meanwhile, restricted by the incompleteness of information and the fuzziness of human thought, hardly anyone can deny that humans usually cannot provide the accurate judgements to the objects. Under such circumstance, fuzzy sets [24], proposed in 1965, have become a useful and powerful tool to express the vagueness of the objects by using a membership degree to represent the superiority of an object. However, with the evolution and complication of the decision making environments, it is noted that the membership degree is often insufficient to portray the uncertainty of things. To overcome the weakness of fuzzy sets, the intuitionistic fuzzy set (IFS) [25-26] was proposed by adding a non-membership degree. IFS describes an object simultaneously from both the superiority and inferiority aspects. The past couple of decades has witnessed the strong development of IFS since it reflects the fuzziness of things more comprehensively and detailedly.

Naturally, the research on decision making in an intuitionistic fuzzy environment has received 
lots of attention, and many decision making methods have been proposed, such as TOPSIS [27], ELECTRE [28], and VIKOR [29], AHP (Analytic Hierarchy Process) [30], WASPAS (weighted aggregated sum product assessment) [31], LINMAP (linear programming techniques for multidimensional analysis of preference) [32-34] etc. As mentioned before, these methods focus only on using numerical values to obtain the decision making results, but ignore a significant character of the decision information, i.e., its distribution. As a result, the decision making results derived by these approaches may lack the dependability. In order to increase the effectiveness and reliability of the decision making methods in an intuitionistic fuzzy environment, in this paper, we take the advantage of the knowledge of thermodynamics to propose a thermodynamic method for the MCDM problems with intuitionistic fuzzy numbers (IFNs).

With the problems in Chinese medical industry (such as the uneven distribution of medical resources, the low efficiency of medical industry, and the unsound health-care system) and the extreme weather conditions emerged in cities across China recently (such as haze and typhoon), the lung diseases in China have increased sharply. Under such circumstances, how to optimize the allocation of medical resources and improve the input-output efficiency of medical care systems are the difficulties and challenges faced by the medical industry of China. Hierarchical medical system, as a significant part of medical decision making support, aims to improve services at county-level and township-level health centers. The effective operation of the hierarchical medical system can reduce the wasting of medical resources and relieve the pressure from the population overcrowding in big hospitals. At present, with the increasing number of people with lung diseases, it is urgent to make the best use of medical resources to confront various lung diseases. Thus, establishing a proper method to assist to classify the patients in different conditions into different levels of 
hospitals is an effective and efficient way to utilize the limited resource and cure more people with lung diseases.

The key to classify patients is the diagnoses of patients. Several doctors usually participate to decide whether or not a patient needs to be provided referral. For the complexity and diversity of the symptoms of lung diseases, the doctors hardly provide the accurate judgements to patients for each symptom. In such cases, the IFNs are useful to be utilized to depict the fuzziness of doctors' assessments. Moreover, in the medical diagnosis process, the quantity and the quality of the data provided by doctors are both significant. On the one hand, the numerical values have directly impact on the diagnosis results. On the other hand, due to that doctors' diagnosis opinions may be different, the quality of the opinions is very critical to decide if the diagnoses should be trusted and utilized. To this end, the thermodynamic method for the MCDM problems with IFNs is a very useful one to be applied to distinguish the quality of doctors' opinions and then assist the decision making in the hierarchical medical system.

The novel aspects of the paper can be summarized as:

(1) The paper introduces the thermodynamics into MCDM and defines the thermodynamic decision making parameters, which link the descriptive characters of physical thermodynamic parameters to the decision making environment.

(2) The paper proposes the thermodynamic method for MCDM with IFNs, which is able not merely to capture the uncertainty of the objects, but also portray the decision making information from both the quantity and quality points of view.

(3) The paper designs the simulations to compare the proposed method withthe intuitionistic fuzzy 
decision making method with weighted averaging operator (WAO) and the intuitionistic fuzzy TOPSIS, which show that the quality of the decision making information is a crucial factor to the decision making results. The conclusions indicate that the proposed method is effective to be utilized in practical decision making problems, especially the uncertain and complicated ones such as medical decision making support.

(4) The paper applies the proposed method to support the hierarchical medical system in West China Hospital, which illustrates the applicability and usefulness of the proposed method. Furthermore, by comparing the results obtained by using other decision making methods to solve the problem, this paper demonstrates that taking the quality (distribution) of the decision making information into account makes the result be more comprehensive and dependable.

The remainder of the paper is organized as follows: Section 2 presents preliminaries related to thermodynamics and IFNs. Section 3 analogizes some definitions about thermodynamic decision making according to physical thermodynamic terms, then proposes a thermodynamic decision making method in intuitionistic fuzzy environment. Section 4 provides the simulative comparisons between the thermodynamic method, the method with WAO and the TOPSIS in an intuitionistic fuzzy environment, and some comparative results are concluded in this section. The application of the proposed method about assisting the hierarchical medical system in West China Hospital is illustrated in Section 5. Some conclusions are listed in Section 6.

\section{Preliminaries}

In this section, we present some basic concepts related to thermodynamics and intuitionistic 
fuzzy number (IFN), which will be used in the next sections.

\subsection{Knowledge about thermodynamics}

Thermodynamics is a subject concerning the interaction between the system and the environment when the state of matter changes. In what follows, we recall the concepts of energy, exergy, entropy, and two basic laws of thermodynamics.

\section{Definition 2.1 [18-20].}

(1) Energy is the ability of objects which can be transferred into others or converted into different forms;

(2) Exergy of a system is the maximum useful work possible during a process that brings the system into equilibrium, when the thermodynamic system is disequilibrium with the environment;

(3) Entropy is a measurement to depict the disorder of the system. It is a parameter to depict the irreversibility in the spontaneous process.

Definition 2.2 [21]. (First law of thermodynamics) The energy can be transformed from one form to another, but the total energy of an isolated system is constant.

Definition 2.3 [22]. (Second law of thermodynamics) The entropy of a system will not decrease with the passing of time.

Based on the above two basic laws of thermodynamics, we can easily find out that, even if the energies of two systems are the same, their exergies and entropies probably are disparate, which can be considered that the quantities of the two systems are equivalent, but their qualities are different. Moreover, for an isolated system, energy is constant. But as the time goes by, the useful work of 
the system decreases and the invalid energy increases, which mean that exergy reduces and entropy increases.

\subsection{Knowledge about IFNs}

Let $\alpha=\left(\mu_{\alpha}, v_{\alpha}\right)$ be an IFN, where $\mu_{\alpha} \in[0,1], v_{\alpha} \in[0,1]$ and $0 \leq \mu_{\alpha}+v_{\alpha} \leq 1 . \mu_{\alpha}$ and $v_{\alpha}$ respectively indicate the membership degree and the non-membership degree. The expression $\pi_{\alpha}=1-\mu_{\alpha}-v_{\alpha}$ is called the hesitant degree [35]. Now we introduce the operational laws for IFNs as follows:

Definition 2.4 [35-37]. Let $\alpha=\left(\mu_{\alpha}, v_{\alpha}\right), \alpha_{1}=\left(\mu_{\alpha_{1}}, v_{\alpha_{1}}\right)$ and $\alpha_{2}=\left(\mu_{\alpha_{2}}, v_{\alpha_{2}}\right)$ be three IFNs, then

(1) $\alpha^{c}=\left(v_{\alpha}, \mu_{\alpha}\right)$;

(2) $\alpha_{1} \oplus \alpha_{2}=\left(\mu_{\alpha_{1}}+\mu_{\alpha_{2}}-\mu_{\alpha_{1}} \mu_{\alpha_{2}}, v_{\alpha_{1}} v_{\alpha_{2}}\right)$;

(3) $\alpha_{1} ! \alpha_{2}=\left(\mu_{\alpha_{1} ! \alpha_{2}}, v_{\alpha_{1} ! \alpha_{2}}\right)$, where

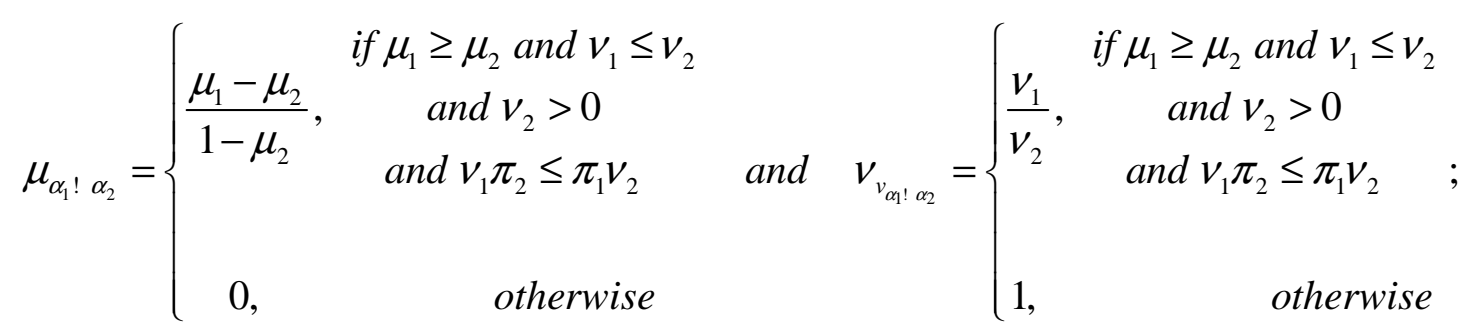

(4) $\lambda \alpha=\left(1-\left(1-\mu_{\alpha}\right)^{\lambda}, v_{\alpha}^{\lambda}\right), \lambda>0$.

Definition 2.5 [35]. The score function and the accuracy function of an IFN are defined as $s(\alpha)=\mu_{\alpha}-v_{\alpha}$ and $h(\alpha)=\mu_{\alpha}+v_{\alpha}$ respectively. Then for two IFNs $\alpha_{1}$ and $\alpha_{2}$ :

(1) If $s\left(\alpha_{1}\right)>s\left(\alpha_{2}\right)$, then $\alpha_{1}>\alpha_{2}$;

(2) If $s\left(\alpha_{1}\right)=s\left(\alpha_{2}\right)$, then

(a) If $h\left(\alpha_{1}\right)>h\left(\alpha_{2}\right)$, then $\alpha_{1}>\alpha_{2}$; 
(b) If $h\left(\alpha_{1}\right)=h\left(\alpha_{2}\right)$, then $\alpha_{1}=\alpha_{2}$.

For two IFNs $\alpha_{1}$ and $\alpha_{2}$, the Hamming distance [38] between them is expressed as:

$$
d\left(\alpha_{1}, \alpha_{2}\right)=\frac{1}{2}\left(\left|\mu_{\alpha_{1}}-\mu_{\alpha_{2}}\right|+\left|v_{\alpha_{1}}-v_{\alpha_{2}}\right|+\left|\pi_{\alpha_{1}}-\pi_{\alpha_{2}}\right|\right)
$$

An aggregation operator, which is effective to be used in the decision making process, is shown as follows:

Theorem 2.1 [36]. Let $\alpha_{j}=\left(\mu_{\alpha_{j}}, v_{\alpha_{j}}\right)(j=1,2, \ldots, n)$ be a collection of IFNs, and $\omega=\left(\omega_{1}, \omega_{2}, \cdots, \omega_{n}\right)^{T}$ is the weight vector of $\alpha_{j}=\left(\mu_{\alpha_{j}}, v_{\alpha_{j}}\right)(j=1,2, \ldots, n)$ with $\omega_{j} \in[0,1]$ $(j=1,2, \cdots, n)$, and $\sum_{j=1}^{n} \omega_{j}=1$. The function IFWA is called the intuitionistic fuzzy weighted averaging (IFWA) operator if

$$
\operatorname{IFWA} A_{\omega}\left(\alpha_{1}, \alpha_{2}, \cdots, \alpha_{n}\right)=\bigoplus_{i=1}^{n} \omega_{i} \alpha_{i}=\omega_{1} \alpha_{1} \oplus \omega_{2} \alpha_{2} \oplus \cdots \oplus \omega_{n} \alpha_{n}=\left(1-\prod_{j=1}^{n}\left(1-\mu_{\alpha_{j}}\right)^{\omega_{j}}, \prod_{j=1}^{n} v_{\alpha_{j}}^{\omega_{j}}\right)
$$

\section{Thermodynamic Decision Making with IFNs}

\subsection{Description of the MCDM problem with IFNs}

Before we present the details about the proposed thermodynamic method for MCDM in an intuitionistic fuzzy environment, we briefly describe the considered problem as below:

A MCDM problem with IFNs is how to find or select the most desirable alternative with respect to a series of criteria, based on the assessment values as IFNs by a group of decision makers (DMs). 
Mathematically, the above MCDM problem can be specified as follows: Let $A=\left\{A_{i} \mid i \in M\right\}$ be the set of $m$ alternatives, and $C=\left\{C_{j} \mid j \in N\right\}$ be the set of $n$ criteria. $l$ DMs are invited to assess the alternatives. The weight vector of criteria given by DM $k$ is $\omega=\left(\omega_{1}^{k}, \omega_{2}^{k}, \ldots, \omega_{n}^{k}\right)^{T}$, where $0 \leq \omega_{j}^{k} \leq 1(j=1,2, \ldots, n) \quad(k=1,2, \ldots, l)$ and $\sum_{j=1}^{n} \omega_{j}^{k}=1$ for all $k=1,2, \ldots, l$. The $k$-th DM also provides his/her assessment value over alternative $A_{i}$ with respect to criterion $C_{j}$ as IFN $\alpha_{i j}^{k}=\left(\mu_{i j}^{k}, v_{i j}^{k}\right)$, where $\mu_{i j}^{k}$ and $v_{i j}^{k}$ are the degrees that the alternative $A_{i}$ satisfies/dissatisfies $C_{j}$ respectively. Then the intuitionistic fuzzy assessment matrixes $R^{k}=\left(r_{i j}^{k}\right)_{m \times n} \quad(k=1,2, \ldots, l)$ provided by the DMs can be expressed as:



\subsection{The thermodynamic method for MCDM with IFNs}

In this subsection, we firstly analogize some definitions about thermodynamic decision making according to physical thermodynamic terms, and then propose a thermodynamic method for the MCDM problems in an intuitionistic fuzzy environment.

Definition 3.1. Decision potential indicates the potential energy of an alternative with respect to a criterion, which can be expressed by the corresponding criterion value.

Definition 3.2. Decision force is defined as the weight of a criterion.

For an assessment value of an alternative with respect to a criterion, we can present its decision potential and the corresponding decision force by a diagram: 


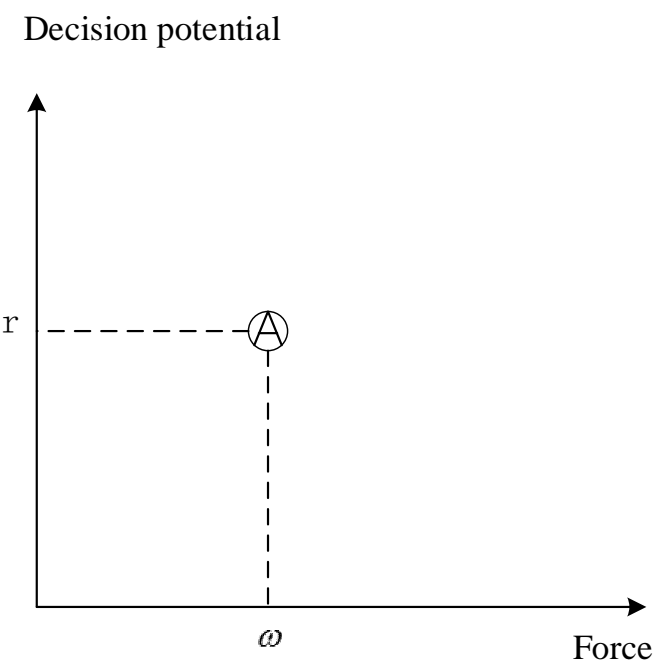

Fig. 1. The decision potential of an alternative and its decision force

Example 3.1. If a DM assesses an object with respect to a criterion as $(0.5,0.2)$, and the weight of the criterion is 0.35 , then the decision potential and the decision force of the object are $(0.5,0.2)$ and 0.35 respectively.

Then, the decision energy of an alternative can be defined:

Definition 3.3. In a state, decision energy is a property of an alternative, which expresses energy that the alternative possesses in the system, which relates to its decision potential and the corresponding decision force:

$$
E=\omega r
$$

Example 3.2. Based on Example 3.1, the decision energy of the object can be obtained:

$$
E=0.35 \otimes(0.5,0.2)=(0.2154,0.5693)
$$

Decision energy is to measure the quantity of the decision information. Next, we conduct the definition of decision work as follows: 
Definition 3.4. When a system changes from state $r_{1}$ to state $r_{2}$, the decision work performed by the system is expressed as:

$$
W=\omega d\left(r_{1}, r_{2}\right)
$$

where $d\left(r_{1}, r_{2}\right)$ is the distance between the decision potentials of two states $r_{1}$ and $r_{2}$.

Example 3.3. To assess an object with respect to a criterion with the decision force as 0.6 , if the original decision potential of the object is $(0.5,0.1)$, after discovering more information, its decision potential is needed to be adjusted as $(0.6,0.3)$, then the decision work in this process is calculated by:

$$
W=0.6 \times \frac{1}{2}(|0.5-0.6|+|0.1-0.3|+|0.4-0.1|)=0.18
$$

Until here, the decision making process in an intuitionistic fuzzy environment can be done by the method with WAO, the TOPSIS and any other decision making methods. Decision energy is commonly used to demonstrate the results of the decision making problems. In other words, we usually make decision in accordance with the quantity of decision potentials. However, it should be noticed that we ignore a significant character that is the quality of the decision potentials. For example, if four DMs assess object 1 as $r_{1}=(0.6,0.6), \quad r_{2}=(0.6,0.6), \quad r_{3}=(0.6,0.4)$ and $r_{4}=(0.6,0.9)$, and object 2 as $r_{1}{ }^{\prime}=(0.6,0.9), \quad r_{2}{ }^{\prime}=(0.6,0.4), \quad r_{3}{ }^{\prime}=(0.6,0.4)$ and $r_{4}{ }^{\prime}=(0.6,0.9)$, then by the WAO under the assumptions of the same weights for all four DMs, we get that the decision energy of object 1 is equal to that of object 2. Nevertheless, these two objects are obviously different. From the distribution of the assessments for the two objects, we know that object 1 is better than object 2 because its decision potentials are more centralized, which is considered to be more reliable. 
In order to depict the quality of decision potentials so as to increase the dependability of the decision making results, below we introduce some concepts:

Definition 3.5. The quality of a decision potential for DM $i$ is measured by the similarity [24] between itself and the mean decision potential among all DMs:

$$
Q_{i}=1-\frac{d\left(r_{i}, \bar{r}\right)}{d\left(r_{i}, \bar{r}\right)+d\left(r_{i}^{c}, \bar{r}\right)}
$$

where $r_{i}^{c}$ is the complementary of $r_{i}$, and $\bar{r}$ is the mean decision potential calculated by the IFWA operator. Especially, if the decision potentials given by the DMs are the same, then $Q_{i}(i=1,2, \ldots, l)=1$, which indicates that the quality of each decision potential is the best due to the highest degree of consistency. .

Definition 3.6. Decision exergy is the maximum effectiveness of decision potentials, which is expressed as:

$$
B=Q E
$$

Decision exergy is able to measure the quality of decision potentials. It can describe the result obtained by a series of decision potentials, which takes the distribution of these decision potentials into account. Considering the common use of entropy to depict the quality from the aspect of instability of a system, we provide as below a definition of decision entropy according to the relationship between exergy and entropy:

Definition 3.7. Decision entropy is to measure the unevenness of decision potentials of an alternative, which is given as:

$$
S=E ! \quad B
$$


If the decision potentials of an alternative provided by the DMs are more centralized, then the decision entropy of the corresponding alternative is smaller. Decision entropy provides a strong and feasible tool to assess the alternatives on both sides of quantity and quality, which makes the decision making results more reliable and credible.

Example 3.4. On the basis of Example 3.2, suppose that the mean decision potential is obtained as $(0.8,0.04)$, then the quality of the decision potential can be calculated as:

$$
Q=1-\frac{d((0.5,0.2),(0.8,0.04))}{d((0.5,0.2),(0.8,0.04))+d((0.2,0.5),(0.8,0.04))}=0.3333
$$

while the decision exergy and the decision entropy of the decision potential are respectively obtained as:

$$
\begin{gathered}
B=Q E=0.3333 \otimes(0.2154,0.5693)=(0.0777,0.8288) \\
S=E ! B=(0.2154,0.5693) !(0.0777,0.8288)=(0.1493,0.6869)
\end{gathered}
$$

As the end of this section, we give the whole process of the thermodynamic method for solving the MCDM problem in an intuitionistic fuzzy environment as follows:

Step 1. Identify alternatives $A_{i}(i=1,2, \ldots, m)$ and criteria $C_{j}(j=1,2, \ldots, n)$ of the MCDM problem, and invite the DMs $D_{k}(k=1,2, \ldots, l)$ to assess the alternatives by providing the intuitionistic fuzzy decision matrixes $R^{k}=\left(r_{i j}^{k}\right)_{m \times n}(k=1,2, \ldots, l)$ :

$$
R^{k}=\left(r_{i j}{ }^{k}\right)_{m \times n}=\left(\begin{array}{cccc}
\left(\mu_{11}^{k}, v_{11}^{k}\right) & \left(\mu_{12}^{k}, v_{12}^{k}\right) & \cdots & \left(\mu_{1 n}^{k}, v_{1 n}^{k}\right) \\
\left(\mu_{21}^{k}, v_{21}^{k}\right) & \left(\mu_{22}^{k}, v_{22}^{k}\right) & \cdots & \left(\mu_{2 n}^{k}, v_{2 n}^{k}\right) \\
\vdots & \vdots & \vdots & \vdots \\
\left(\mu_{m 1}^{k}, v_{m 1}^{k}\right) & \left(\mu_{m 2}^{k}, v_{m 2}^{k}\right) & \cdots & \left(\mu_{m n}^{k}, v_{m n}^{k}\right)
\end{array}\right) \quad(k=1,2, \ldots, l)
$$

Step 2. Ask DMs $D_{k}(k=1,2, \ldots, l)$ to determine the weights of the criteria and then obtain 
$\omega_{j}^{k}(j=1,2, \ldots, n) \quad(k=1,2, \ldots, l)$, which indicates that the $k$-th $\mathrm{DM}$ assigns weight $\omega_{j}^{k}$ to the $j$-th criterion.

Step 3. Calculate the decision energy matrixes $E^{k}(k=1,2, \ldots, l)$ of each DM:

$$
E^{k}=\left(\begin{array}{cccc}
\omega_{1}^{k} r_{11}^{k} & \omega_{2}^{k} r_{12}^{k} & \cdots & \omega_{n}^{k} r_{1 n}^{k} \\
\omega_{1}^{k} r_{21}^{k} & \omega_{2}^{k} r_{22}^{k} & \cdots & \omega_{n}^{k} r_{2 n}^{k} \\
\vdots & \vdots & \vdots & \vdots \\
\omega_{1}^{k} r_{m 1}^{k} & \omega_{2}^{k} r_{m 2}^{k} & \cdots & \omega_{n}^{k} r_{m n}^{k}
\end{array}\right)(k=1,2, \ldots, l)
$$

Step 4. Establish the quality matrixes $Q^{k}(k=1,2, \ldots, l)$ of each DM:

$$
Q^{k}=\left(\begin{array}{cccc}
1-\frac{d\left(r_{11}^{k}, \bar{r}_{1}^{k}\right)}{d\left(r_{11}^{k}, \bar{r}_{1}^{k}\right)+d\left(\left(r_{11}^{k}\right)^{c}, \bar{r}_{1}^{k}\right)} & 1-\frac{d\left(r_{21}^{k}, \bar{r}_{2}^{k}\right)}{d\left(r_{21}^{k}, \bar{r}_{2}^{k}\right)+d\left(\left(r_{21}^{k}\right)^{c}, \bar{r}_{2}^{k}\right)} & \cdots & 1-\frac{d\left(r_{n 1}^{k}, \bar{r}_{n}^{k}\right)}{d\left(r_{n 1}^{k}, \bar{r}_{n}^{k}\right)+d\left(\left(r_{n 1}^{k}\right)^{c}, \bar{r}_{n}^{k}\right)} \\
1-\frac{d\left(r_{12}^{k}, \bar{r}_{1}^{k}\right)}{d\left(r_{12}^{k}, \bar{r}_{1}^{k}\right)+d\left(\left(r_{12}^{k}\right)^{c}, \bar{r}_{1}^{k}\right)} & 1-\frac{d\left(r_{22}^{k}, \bar{r}_{2}^{k}\right)}{d\left(r_{22}^{k}, \bar{r}_{2}^{k}\right)+d\left(\left(r_{22}^{k}\right)^{c}, \bar{r}_{2}^{k}\right)} & \cdots & 1-\frac{d\left(r_{n 2}^{k}, \bar{r}_{n}^{k}\right)}{d\left(r_{n 2}^{k}, \bar{r}_{n}^{k}\right)+d\left(\left(r_{n 2}^{k}\right)^{c}, \bar{r}_{n}^{k}\right)} \\
\vdots & \vdots & \vdots & \vdots \\
1-\frac{d\left(r_{1 m}^{k}, \bar{r}_{1}^{k}\right)}{d\left(r_{1 m}^{k}, \bar{r}_{1}^{k}\right)+d\left(\left(r_{1 m}^{k}\right)^{c}, \bar{r}_{1}^{k}\right)} & 1-\frac{d\left(r_{m n}^{k}, \bar{r}_{n}^{k}\right)}{d\left(r_{2 m}^{k}, \bar{r}_{2}^{k}\right)+d\left(\left(r_{2 m}^{k}\right)^{c}, \bar{r}_{2}^{k}\right)} & \cdots & 1-\frac{1\left(r_{m n}^{k}, \bar{r}_{n}^{k}\right)+d\left(\left(r_{m n}^{k}\right)^{c}, \bar{r}_{n}^{k}\right)}{\left.d^{k}\right)}
\end{array}\right),
$$

$$
k=1,2, \ldots, l
$$

where $\bar{r}_{1}^{k}=\left(1-\prod_{j=1}^{n}\left(1-\mu_{1 j}^{k}\right)^{\omega_{j}}, \prod_{j=1}^{n}\left(v_{1 j}^{k}\right)^{\omega_{j}}\right)$.

Step 5. Construct the decision exergy matrixes $B^{k}=\left[Q_{i j}^{k} E_{i j}^{k}\right]_{m \times n}(k=1,2, \ldots, l)$ of each DM.

Step 6. Obtain the averaging decision energy and the averaging decision exergy of the $i$-th alternative in regard to the $k$-th DM:

$$
\bar{E}_{i}^{k}=\frac{1}{n}\left(E_{i 1}^{k} \oplus E_{i 2}^{k} \oplus \cdots \oplus E_{i n}^{k}\right)
$$




$$
\bar{B}_{i}^{k}=\frac{1}{n}\left(B_{i 1}^{k} \oplus B_{i 2}^{k} \oplus \cdots \oplus B_{i n}^{k}\right)
$$

Step 7. Calculate the decision energy and the decision exergy of each alternative:

$$
\begin{aligned}
& E_{i}=\frac{1}{k}\left(\bar{E}_{i}^{1} \oplus \bar{E}_{i}^{2} \oplus \cdots \oplus \bar{E}_{i}^{l}\right) \\
& B_{i}=\frac{1}{k}\left(\bar{B}_{i}^{1} \oplus \bar{B}_{i}^{2} \oplus \cdots \oplus \bar{B}_{i}^{l}\right)
\end{aligned}
$$

Step 8. Determine the decision entropy of each alternative:

$$
S_{i}=E_{i} ! B_{i}
$$

Step 9. Obtain the scores of each decision entropy $s\left(S_{i}\right)$ by Definition 2.5, and then rank the alternatives. The smaller score of the decision entropy, the better the alternative.

Remark 1. The thermodynamic method not only draws on the numerical values of the decision potentials, but also take the dispersion degree of them that can depict the reliability of the decision potentials. This method organically combines the quantity and the quality of the decision potentials, which provides a more visual and rational ranking of the alternatives.

Remark 2. The thermodynamic method has the ability to handle the MCDM problems of different weight vectors of criteria provided by each DM, which is of strong applicability in real world. As we all know, people usually have different viewpoints and opinions with each other, and thus in group decision making, the weights of criteria provided by the DMs are often not the same. The majority of the traditional methods are only adept in dealing with one weight vector of the criteria, which means that we must aggregate the weight vectors into a weight vector. Inevitably, we will lose some original information of these weight vectors in data processing. Therefore, the thermodynamic method is very useful and effective to consider all DMs' opinions, including the 
weights of criteria, the decision information of alternatives and so on.

Remark 3. The intuitionistic fuzzy information is suitable to be applied to actual circumstances, where it can depict the superior and inferior aspects of an object. Considering the uncertainty of an object and the limitation of the human's brain, there always exists fuzziness in daily events, and thus IFNs are available to depict such vagueness. By taking the advantage of IFNs in expressing the opinions of the DMs and the reliability of the thermodynamic method in depicting both the quantity and quality of the decision information, the thermodynamic method for MCDM with IFNs is perfect and very practical in tackling the decision making problems in various fields.

\section{Comparative Analysis}

As mentioned before, the thermodynamic method for MCDM with IFNs is capable of combining the quantity and quality of the decision information in a decision making process, while other commonly used decision making methods cannot achieve the same due to that they only take the quantity of the decision information into account. Apparently, by the two types of methods, the decision making results may be different. Considering the popularity of the WAO and TOPSIS methods in practical decision making problems, which both only consider the quantity of the decision information, we will make the comparisons between the proposed method, the intuitionistic fuzzy decision making method with WAO and the intuitionistic fuzzy TOPSIS in this section.

We conduct simulations to provide two comparative analyses: 1 . Compare the results obtained by three decision making methods, including the thermodynamic method that concerns the quantity and the quality of data, the intuitionistic fuzzy decision making method with WAO and the 
intuitionistic fuzzy TOPSIS that focus on the quantity of data, and then provide the comparative conclusions; 2. Judge whether the comparative conclusions obtained from the first comparative analysis can be affected by the number of criteria and the number of the alternatives.

\subsection{Comparisons of decision making results derived by three decision making methods}

Before comparing the decision making results among three decision making methods, below we briefly present the process of the method with WAO and the TOPSIS in an intuitionistic fuzzy environment:

Part 1. The process of using the intuitionistic fuzzy decision making method with WAO [36]

Step 1. According to the weights of the DMs, we use Eq. (2.2) to aggregate the decision matrixes provided by each DM into an overall decision matrix.

Step 2. According to the weight of each criterion, we use Eq. (2.2) to aggregate the elements in the overall decision matrix to obtain the final results of alternatives.

Step 3. Rank the alternatives based on the final results. The bigger the final results, the better the alternative.

Part 2. The process of the intuitionistic fuzzy TOPSIS [27]

Step 1. According to the weights of DMs, we aggregate the decision matrixes provided by each DM into an overall decision matrix by Eq. (2.2).

Step 2. Identify the intuitionistic fuzzy positive ideal solution (PIS) and the intuitionistic fuzzy negative ideal solution (NIS) of the overall decision matrix by the following formulas [27]: 


$$
P I S=\left\{C_{j}, \max _{i}\left\langle s\left(C_{j}\left(x_{i}\right)\right)\right\rangle \mid j=1,2, \ldots, n\right\}=\left\{\left\langle C_{1},\left(\mu_{1}^{+}, v_{1}^{+}\right)\right\rangle,\left\langle C_{2},\left(\mu_{2}^{+}, v_{2}^{+}\right)\right\rangle, \cdots,\left\langle C_{j},\left(\mu_{n}^{+}, v_{n}^{+}\right)\right\rangle\right\}
$$

and

$$
N I S=\left\{C_{j}, \min _{i}\left\langle s\left(C_{j}\left(x_{i}\right)\right)\right\rangle \mid j=1,2, \ldots, n\right\}=\left\{\left\langle C_{1},\left(\mu_{1}^{-}, v_{1}^{-}\right)\right\rangle,\left\langle C_{2},\left(\mu_{2}^{-}, v_{2}^{-}\right)\right\rangle, \cdots,\left\langle C_{j},\left(\mu_{n}^{-}, v_{n}^{-}\right)\right\rangle\right\}
$$

Step 3. Calculate the distance between alternative $A_{i}$ and the intuitionistic fuzzy PIS [27] in the overall decision matrix by $d\left(A_{i}, P I S\right)=\sum_{j=1}^{n} \omega_{j} d\left(C_{j}\left(A_{i}\right), C_{j}(P I S)\right)$, and the distance between alternative $A_{i}$ and the intuitionistic fuzzy NIS [27] by $d\left(A_{i}, N I S\right)=\sum_{j=1}^{j} \omega_{j} d\left(C_{j}\left(A_{i}\right), C_{j}(N I S)\right)$, where $d\left(C_{j}\left(A_{i}\right), C_{j}(P I S)\right)$ and $d\left(C_{j}\left(A_{i}\right), C_{j}(N I S)\right)$ are obtained by Eq. (2.1).

Step 4. Calculate the relative closeness (RC) indexes for each alternative [27]:

$$
R C=\frac{d\left(A_{i}, N I S\right)}{d\left(A_{i}, P I S\right)+d\left(A_{i}, N I S\right)}
$$

Step 5. Rank the alternatives according to the values of RC. The bigger the value of RC, the better the alternative.

In this simulative analysis, we randomly generate the MCDM problems with IFNs for 1000 times, which aim to select a desirable alternative, and then handle them by the thermodynamic method, the method with WAO and the TOPSIS. Without loss of generality, we select the MCDM problems of five criteria, five alternatives and five DMs. In the simulation process, we record the optimal selection with each decision making method under each MCDM problem, and then compare the decision making results among these methods.

The comparative results derived from Matlab are shown in the following figure: 


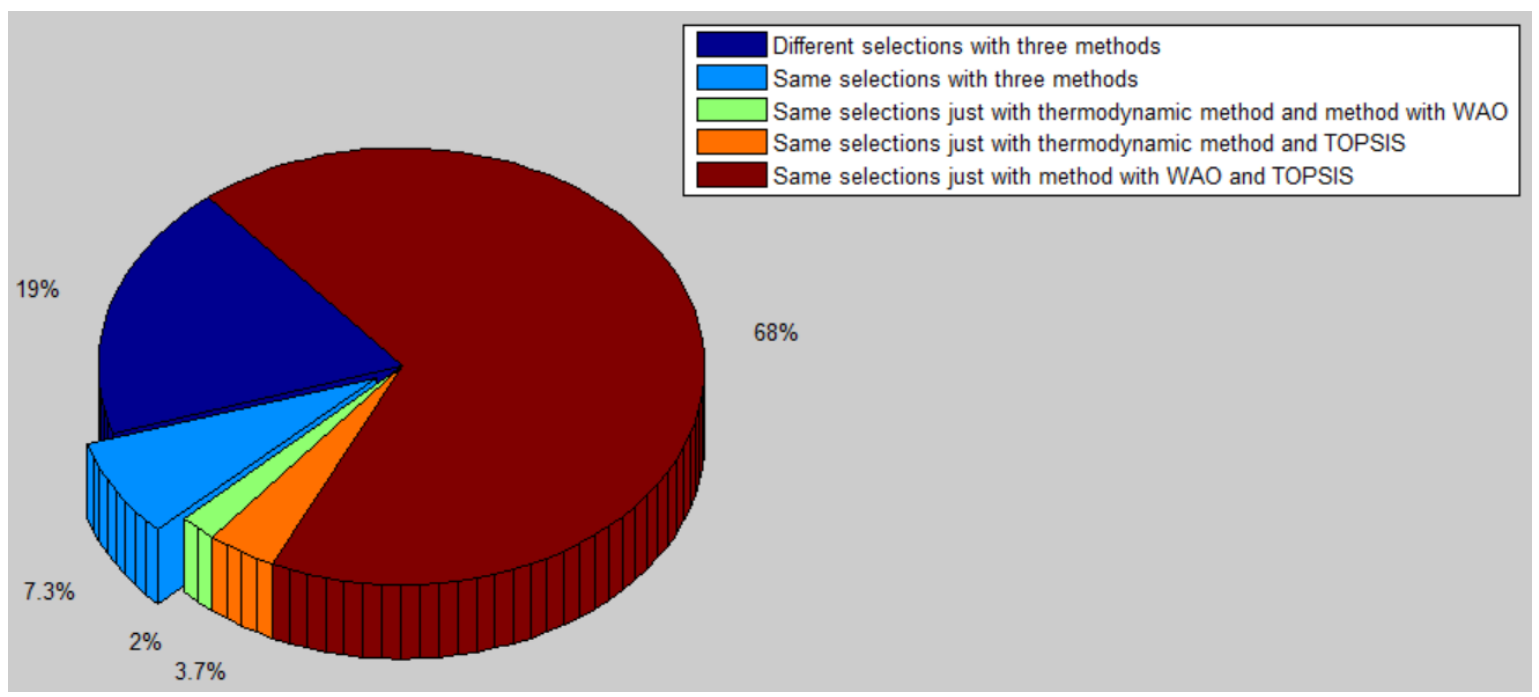

Fig. 2. The comparative results derived by three decision making methods

In Fig. 2, the items represented by each color have been marked, and we can obtain some visualized conclusions: Among the 1000 simulations, the percentage of the optimal selections, derived from the three decision making methods which are different, is $19 \%$, but yet the percentage of reaching the same optimal selection is $7.3 \%$. According to the comparisons of the optimal results between any two decision making methods, the ratios of the same selections obtained by the thermodynamic method and the method with WAO, the thermodynamic method and the TOPSIS, the method with WAO and the TOPSIS are respectively $2 \%, 3.7 \%$, and $68 \%$. To this end, some comparative conclusions can be drawn as follows:

(1) From the above figure, the ratio of obtaining the same optimal selections with the three decision making methods is several times greater than the ratio of reaching the same decision making results with the thermodynamic method and the method with WAO (or the TOPSIS). That is to say, if the optimal selections derived from the thermodynamic method are the same as the selection derived from the method with WAO (or the TOPSIS), then it is probably that these three decision making methods would derive the same decision making result. 
(2) The optimal selections with the thermodynamic method are the most different from the optimal selections obtained by the method with WAO and the TOPSIS. But the decision making results derived from the method with WAO and the TOPSIS are the mostly same. The essential difference between the thermodynamic method and the other two methods is that the thermodynamic method considers another important aspect: the quality of the decision information, which is the internal structure (or internal distribution) of the decision information. These simulation results show thattaking the quality of the decision information into account often changes the decision making results, which indicates that the quality of decision information is a very significant character we cannot ignore.

\subsection{Sensitive analysis related to decision making results derived by three decision making methods}

In this subsection, we endeavor to investigate whether the simulative results in Fig. 2 can be influenced by the number of criteria or the number of alternatives. The simulation tests are designed as follows:

We randomly generate the MCDM problems of five attributes with IFNs for 1000 times and let the number of criteria vary from 3 to 10 . Then the optimal or desired alternative is selected by the thermodynamic method, the method with WAO and the TOPSIS respectively. After that, we record the number of times when the optimal selections with any two decision making methods are the same. Moreover, we randomly conduct other 1000 times simulations for the MCDM problems of five criteria with IFNs, and let the number of alternatives vary from 3 to 10 , and then dispose these MCDM problems like before. The simulative results can be shown in Figs. 3-5. 


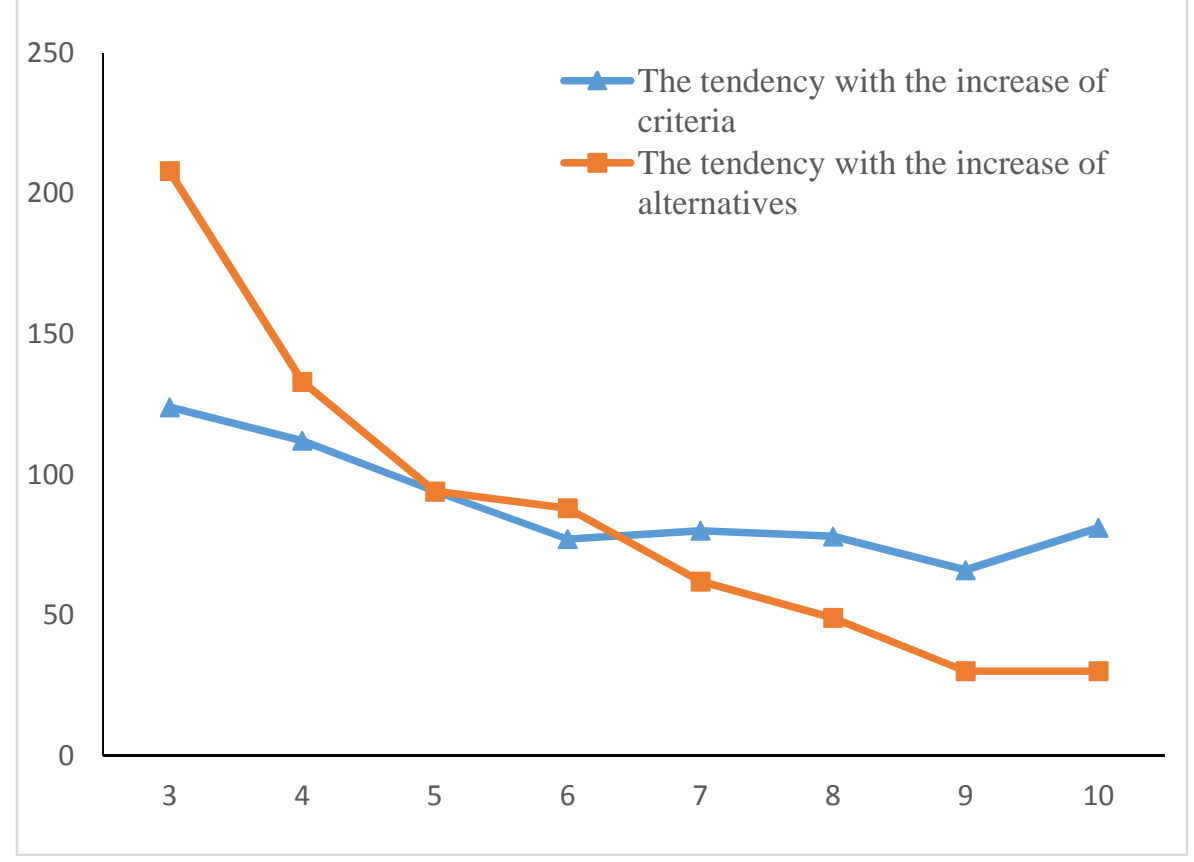

Fig. 3. Same selections with the thermodynamic method and the method with WAO

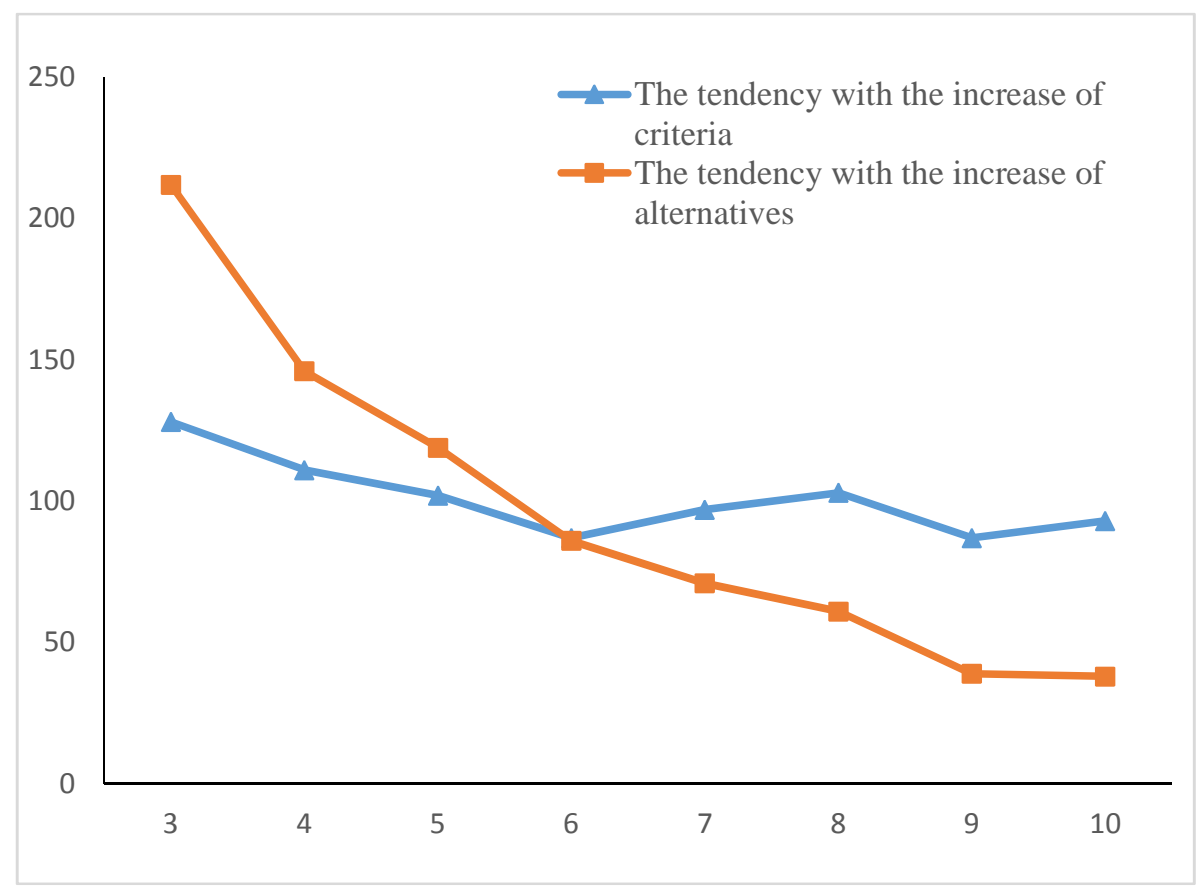

Fig. 4. Same selections with the thermodynamic method and the TOPSIS 


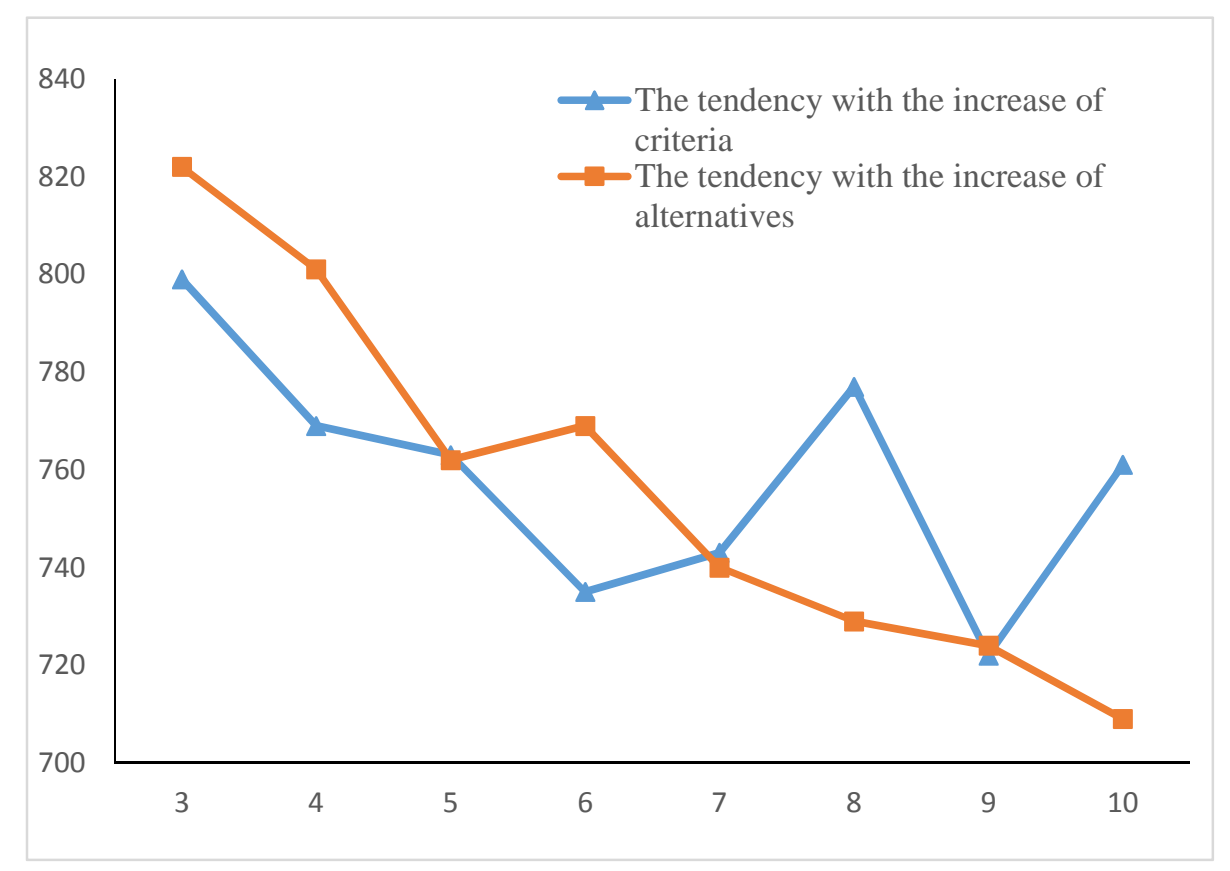

Fig. 5. Same selections with the method with WAO and the TOPSIS

From the above figures, we can make some conclusions below:

(1) The number of times for getting the same optimal selections with any two decision making methods is significantly affected by the number of alternatives. Increasing the number of alternatives leads to the intensifying of the complexity of the structure between alternatives, which makes the decision making systems to be diversified.

(2) Although increasing the number of criteria can add the number of criteria values for each alternative, it hardly affects any two decision making methods to obtain the same optimal selections.

(3) With the increasing of the number of alternatives, the tendency of getting the same optimal selections with the thermodynamic method and the method with WAO (or the TOPSIS) is still declining. But this decreasing tendency between the method with WAO and the TOPSIS is unstable. This indicates that the thermodynamic method is more sensitive to the number of 
alternatives than the other two methods.

\section{Case Analysis}

\subsection{Applications in assisting the hierarchical medical system with the proposed method}

In the past few years, the problems in Chinese medical industry, such as the uneven distribution of medical resource, the low efficiency of medical industry, the unsound health-care system, cause the difficulty and the high cost of patients' treatments and such an issue is becoming more and more serious.

Recently, with the economic globalization of the twenty-first century, people living standard rises ceaselessly. Meanwhile, the conflict between environmental issues and human development is also sharpening. Since 2013, the extreme weather conditions, such as haze and typhoon, have emerged among the cities across China . Air quality indexes in these cities grossly exceed standard. People's health has also been adversely affected. The research results of the global burden of diseases published in the journal The Lancet indicate: Among the risk factors which affect the burden of diseases, outdoor air particulate pollution has reached as high as fourth in China, and about $20 \%$ causative factors in lung cancer is related to air pollution. Due to the increasingly prominent environmental issues, the operations of the medical industry in China is facing a severe test.

During the process of deteriorating weather conditions, on the horizon is the high incidence of lung disease. Hardly can anyone deny the fact that nowadays the number of the sick is increasing with appalling rapidity of the weather conditions. However, due to the uneven distribution of 
medical resource in China, the medical facilities and care conditions in big hospitals are much better than other hospitals. Therefore, citizens are much more willing to seek medical advices and treatments in big hospitals. West China Hospital, which is one of the top hospitals in China, possesses the advanced medical equipment and medical resource. As such, the past couple of decades witnessed the increasing number of the patients, which far excesses the capacity of West China Hospital to handle.

Under such a circumstance, the hierarchical medical system, which aims to classify the difficulty of the treatment according to the disease of the light, heavy, slow, and urgent, and then let the different levels of medical institutions undertake the different kinds of diseases, is introduced as an efficient way to release the pressure of the number of patients in West China Hospital. Fundamentally, how to classify the different degrees of diseases is a critical problem in the hierarchical medical system.

As mentioned before, the issue of lung diseases in this day and age has triggered great attention, which is of great threat and potential impact on the health of humans. Therefore, in this case analysis, we focus on assisting the hierarchical medical system for lung diseases, which include chronic obstructive pulmonary, lung cancer, lower respiratory infection, etc. According to the symptoms of the lung diseases, we consider the following factors associated with the diagnoses for each patient, who is possibly infected with lung diseases:

- The signs of the body. This criterion is a significant indicator to judge the present performance of the body. The signs include the level of heart rate, the level of blood pressure, the level of glucose and others, which are the indications for the conditions. More un-normal signs are 
assigned a smaller judgement value.

- The level of body temperature. The body temperature is a clinical manifestation of some kinds of diseases. Shivering and hyperthermia are two classical symptoms of pneumonia, which can be caused by a majority of lung diseases. More un-normal body temperature are assigned a smaller judgement value.

- The frequency of cough. The lung diseases frequently cause the bronchial damage, which is easy to produce cough. The symptoms like irritable cough, chronic cough, etc., are helpful to determine the elementary means of diagnosis. The higher the frequency of cough are assigned a smaller judgement value.

- The frequency of hemoptysis. There is an array of blood vessels in the lung, furthermore, the tumor in the lung also contains many blood vessels. The cough may damage blood vessels on the surface layer of the lung and tumor. Different degrees of the hemoptysis are a crucial indicator for judging the state of an illness. The higher the frequency of hemoptysis are assigned a smaller judgement value.

- Extra-pulmonary manifestations of lung diseases. Some patients with early lung diseases may don't manifest the symptoms of the lung diseases mentioned above, but they have other symptoms like arthralgia, and arthrocele. Therefore, these manifestations should also be considered in the diagnose process. More extra-pulmonary manifestations are assigned a smaller judgement value.

According to the primary diagnosis which considers these five criteria, the conditions and the critical degrees of patients can be judged. With these judgements, patients are decided to be 
distributed to which levels and types of hospitals. As for the West China Hospital, the emergency patients should receive treatment in itself. The patients with the less serious conditions should be treated in the second-tier hospitals. Other patients with light illness can seek medical advice and treatment at local hospitals.

In this case analysis, we invite three doctors $\left\{D_{1}, D_{2}, D_{3}\right\}$ with the same weights to diagnose diseases. The three doctors are experts in internal medicine diseases at West China Hospital, one of the best research hospitals in China. All the three doctors have more than 10(?) years experiences in respiration medicine and possess deep diagnostic knowledge of bronchial asthma, pneumothorax, chronic obstructive pulmonary disease, and other respiration diseases . The considered criteria being mentioned above are denoted as $C_{j}(j=1,2,3,4,5)$. The weight vector of these criteria is provided by the doctors as $\boldsymbol{\omega}=(0.25,0.15,0.25,0.25,0.1)^{T}$. Suppose that there are five patients $P_{i}(i=1,2,3,4,5)$ with lung diseases, who are needed to be diagnosed and distributed. As mentioned in the Introduction section, the thermodynamic method for MCDM with IFNs is a proper method to be applied to assist the hierarchical medical system. Therefore, this method should be utilized here. The judgements are represented by the IFNs, which can fully express the uncertainty of the objects. Then the judgements for the patients with respect to the symptoms provided by the three doctors are listed below:

$$
R^{1}=\left(\begin{array}{lllll}
(0.7,0.2) & (0.7,0.2) & (0.4,0.3) & (0.6,0.1) & (0.8,0.1) \\
(0.5,0.1) & (0.6,0.2) & (0.5,0.2) & (0.5,0.1) & (0.9,0.1) \\
(0.4,0.4) & (0.4,0.3) & (0.5,0.3) & (0.4,0.2) & (0.7,0.1) \\
(0.8,0.1) & (0.7,0.2) & (0.6,0.2) & (0.6,0.2) & (0.9,0.1) \\
(0.5,0.4) & (0.5,0.3) & (0.4,0.4) & (0.4,0.3) & (0.6,0.2)
\end{array}\right)
$$




$$
\begin{aligned}
& R^{2}=\left(\begin{array}{lllll}
(0.7,0.2) & (0.8,0.2) & (0.5,0.4) & (0.6,0.3) & (0.7,0.1) \\
(0.6,0.1) & (0.6,0.2) & (0.7,0.2) & (0.6,0.2) & (0.8,0.1) \\
(0.5,0.4) & (0.3,0.2) & (0.6,0.3) & (0.6,0.3) & (0.7,0.1) \\
(0.6,0.1) & (0.7,0.3) & (0.6,0.2) & (0.6,0.1) & (0.8,0.1) \\
(0.5,0.3) & (0.5,0.3) & (0.5,0.4) & (0.5,0.4) & (0.7,0.2)
\end{array}\right) \\
& R^{3}=\left(\begin{array}{llllll}
(0.6,0.1) & (0.6,0.2) & (0.3,0.3) & (0.6,0.2) & (0.8,0.1) \\
(0.7,0.2) & (0.5,0.3) & (0.7,0.2) & (0.7,0.3) & (0.7,0.1) \\
(0.2,0.3) & (0.6,0.3) & (0.5,0.2) & (0.6,0.3) & (0.7,0.1) \\
(0.6,0.1) & (0.6,0.1) & (0.7,0.2) & (0.6,0.1) & (0.8,0.1) \\
(0.6,0.4) & (0.6,0.3) & (0.5,0.4) & (0.4,0.3) & (0.6,0.3)
\end{array}\right)
\end{aligned}
$$

From the above information, the process of obtaining the overall results can be presented as follows:

Step 1. Calculate the decision energy matrixes $E^{k}(k=1,2,3)$ of each DM by Eq. (3.8):

$$
\begin{gathered}
E^{1}=\left(\begin{array}{llllll}
(0.2599,0.6687) & (0.1652,0.7855) & (0.1199,0.7401) & (0.2047,0.5623) & (0.1487,0.7943) \\
(0.1591,0.5623) & (0.1284,0.7855) & (0.1591,0.6687) & (0.1591,0.5623) & (0.2057,0.7943) \\
(0.1199,0.7953) & (0.0738,0.8348) & (0.1591,0.7401) & (0.1199,0.6687) & (0.1134,0.7943) \\
(0.3313,0.5623) & (0.1652,0.7855) & (0.2047,0.6687) & (0.2047,0.6687) & (0.2057,0.7943) \\
(0.1591,0.7953) & (0.0987,0.9348) & (0.1199,0.7953) & (0.1199,0.7401) & (0.0876,0.8513)
\end{array}\right) \\
E^{2}=\left(\begin{array}{llllll}
(0.2599,0.6687) & (0.2145,0.7855) & (0.1591,0.7953) & (0.2047,0.7401) & (0.1134,0.7943) \\
(0.2047,0.5623) & (0.1284,0.7855) & (0.2599,0.6687) & (0.2047,0.6687) & (0.1487,0.7943) \\
(0.1591,0.7953) & (0.0521,0.7855) & (0.2047,0.7401) & (0.2047,0.7401) & (0.1134,0.7943) \\
(0.2047,0.5623) & (0.1652,0.8348) & (0.2047,0.6687) & (0.2047,0.5623) & (0.1487,0.7943) \\
(0.1591,0.7401) & (0.0987,0.8348) & (0.1591,0.7953) & (0.1591,0.7953) & (0.1134,0.8513)
\end{array}\right) \\
E^{3}=\left(\begin{array}{llllll}
(0.2047,0.5623) & (0.1284,0.7855) & (0.0853,0.7401) & (0.2047,0.6687) & (0.1487,0.7943) \\
(0.2599,0.6687) & (0.0987,0.8348) & (0.2599,06687) & (0.2599,0.7401) & (0.1134,0.7943) \\
(0.0543,0.7401) & (0.1284,0.8348) & (0.1591,0.6687) & (0.2047,0.7401) & (0.1134,0.7943) \\
(0.2047,0.5623) & (0.1284,0.7079) & (0.2599,0.6687) & (0.2047,0.5623) & (0.1487,0.7943) \\
(0.2047,0.7953) & (0.1284,0.8348) & (0.1591,0.7953) & (0.1199,0.7401) & (0.0876,0.8866)
\end{array}\right)
\end{gathered}
$$


Step 2. Based on the decision energy matrixes, the mean decision energy of each DM can be obtained as:

$$
\begin{aligned}
& \bar{r}^{1}=[(0.6319,0.1737),(0.5883,0.1320),(0.4651,0.2610),(0.7195,0.1569),(0.4644,0.3326)]^{T} \\
& \bar{r}^{2}=[(0.6553,0.2456),(0.6527,0.1569),(0.5531,0.2718),(0.6425,0.1402),(0.5249,0.3326)]^{T} \\
& \bar{r}^{3}=[(0.5707,0.1737),(0.6761,0.2195),(0.5113,0.2429),(0.6527,0.1189),(0.5319,0.3464)]^{T}
\end{aligned}
$$

then the quality matrixes $Q^{k}(k=1,2,3)$ of each DM are established as follows:

$$
\begin{aligned}
Q^{1} & =\left(\begin{array}{lllll}
0.8478 & 0.8478 & 0.5587 & 0.8344 & 0.7884 \\
0.8024 & 0.8545 & 0.8147 & 0.8024 & 0.7113 \\
0.5000 & 0.7172 & 0.7639 & 0.6777 & 0.6515 \\
0.8888 & 0.9265 & 0.8129 & 0.8129 & 0.8046 \\
0.6190 & 0.8244 & 0.5000 & 0.6288 & 0.6634
\end{array}\right) \\
Q^{2} & =\left(\begin{array}{llllll}
0.9090 & 0.7931 & 0.6218 & 0.8653 & 0.7923 \\
0.8345 & 0.8957 & 0.8573 & 0.8957 & 0.8136 \\
0.6403 & 0.5208 & 0.8137 & 0.8137 & 0.7251 \\
0.8676 & 0.7204 & 0.8849 & 0.8676 & 0.8073 \\
0.7963 & 0.7963 & 0.7130 & 0.7130 & 0.6772
\end{array}\right) \\
Q^{3} & =\left(\begin{array}{lllll}
0.8647 & 0.8846 & 0.5000 & 0.8846 & 0.7321 \\
0.9526 & 0.6811 & 0.9526 & 0.8215 & 0.8283 \\
0.4495 & 0.7100 & 0.8518 & 0.7100 & 0.7078 \\
0.8853 & 0.8853 & 0.8190 & 0.8853 & 0.8222 \\
0.6758 & 0.7884 & 0.7413 & 0.5653 & 0.7884
\end{array}\right)
\end{aligned}
$$

Step 3. Construct the exergy matrixes $B^{k}=\left(Q_{i j}^{k} E_{i j}^{k}\right)_{m \times n}(k=1,2,3)$ of each DM:

$$
B^{1}=\left(\begin{array}{lllll}
(0.2252,0.7110) & (0.1420,0.8149) & (0.0724,0.8376) & (0.1740,0.6186) & (0.1192,0.8340) \\
(0.1298,0.6301) & (0.1108,0.8136) & (0.1317,0.7205) & (0.1298,0.6301) & (0.1511,0.8489) \\
(0.0619,0.8918) & (0.0535,0.8785) & (0.1240,0.7946) & (0.0829,0.7614) & (0.0754,0.8607) \\
(0.3007,0.5995) & (0.1541,0.7996) & (0.1699,0.7210) & (0.1699,0.7210) & (0.1691,0.8309) \\
(0.1017,0.8678) & (0.0821,0.8617) & (0.0619,0.8918) & (0.0772,0.8276) & (0.0590,0.8987)
\end{array}\right)
$$




$$
B^{2}=\left(\begin{array}{lllll}
(0.2394,0.6937) & (0.1742,0.8258) & (0.1021,0.8673) & (0.1798,0.7707) & (0.0910,0.8332) \\
(0.1740,0.6185) & (0.1158,0.8055) & (0.2274,0.7083) & (0.1855,0.6974) & (0.1227,0.8392) \\
(0.1050,0.8636) & (0.0275,0.8819) & (0.1701,0.7828) & (0.1701,0.7828) & (0.0836,0.8462) \\
(0.1802,0.6069) & (0.1220,0.8780) & (0.1835,0.7004) & (0.1802,0.6069) & (0.1218,0.8304) \\
(0.1289,0.7869) & (0.0795,0.8661) & (0.1162,0.8493) & (0.1162,0.8493) & (0.0783,0.8967)
\end{array}\right)
$$

$$
B^{3}=\left(\begin{array}{llllll}
(0.1797,0.6079) & (0.1145,0.8077) & (0.0436,0.8603) & (0.1834,0.7005) & (0.1111,0.8449) \\
(0.2493,0.6816) & (0.0684,0.8843) & (0.2493,0.6816) & (0.2191,0.7809) & (0.0949,0.8264) \\
(0.0248,0.8735) & (0.0930,0.8796) & (0.1372,0.7098) & (0.1501,0.8076) & (0.0817,0.8496) \\
(0.1836,0.6007) & (0.1146,0.7366) & (0.2185,0.7193) & (0.1836,0.6007) & (0.1239,0.8275) \\
(0.1434,0.8566) & (0.1027,0.8673) & (0.1206,0.8438) & (0.0696,0.8435) & (0.0697,0.9094)
\end{array}\right)
$$

Step 4. By Eqs. (3.10)-(3.14), the decision making results can be listed in the following table.

Table 1. The decision making results with the thermodynamic method with IFNs

\begin{tabular}{ccccc}
\hline & Decision energy & Decision exergy & Decision entropy & Conditions of patients \\
\hline Patient 1 & $(0.1764,0.7211)$ & $(0.1452,0.7703)$ & $(0.0365,0.9360)$ & 3 \\
Patient 2 & $(0.1852,0.6980)$ & $(0.1592,0.7389)$ & $(0.0310,0.9446)$ & 1 \\
Patient 3 & $(0.1334,0.7628)$ & $(0.0972,0.8292)$ & $(0.0401,0.9199)$ & 5 \\
Patient 4 & $(0.2006,0.6727)$ & $(0.1730,0.7123)$ & $(0.0333,0.9445)$ & 2 \\
Patient 5 & $(0.1322,0.8046)$ & $(0.0942,0.8606)$ & $(0.0420,0.9349)$ & 4 \\
\hline
\end{tabular}

A smaller value of decision entropy indicates the better condition of the patient. Thus, the 
ranking about the conditions of patients can be listed in Table 1, and the patients can be classified according to the ranking. Patient 3 is of the worst condition, who should be distributed in West China Hospital. Based on the availability of the accommodations for patients in each hospital, other four patients can be arranged in different levels of hospitals.

If we only consider the quantity of decision making information provided by the three doctors, then by the decision energy of each patient in Table 1, the overall values of the patients indicate that Patient 5's condition is the most critical, and he/she should be treated in West China Hospital. This occurs because the judgements provided by the three doctors for Patient 3 are more concentrated and steady than Patient 5.

\subsection{Comparison analysis with other decision making methods}

In this subsection, we deal with the problem described in Section 5.1 by the method with WAO [36] and the TOPSIS [27] for MCDM in intuitionistic fuzzy environment, and then compare the decision making results among the three decision making methods.

By Eq. (2.2), we can obtain a ranking of the patients by the intuitionistic fuzzy decision making method with WAO [36]. Meanwhile, according the intuitionistic fuzzy TOPSIS proposed in Ref. [27], we can also obtain another ranking. The results derived from the three decision making methods are collected in Table 2.

Table 2. The decision making results with the three decision making methods

\begin{tabular}{l|l|l|l}
\hline & The thermodynamic method & The method with WAO & The TOPSIS \\
\hline & Final results $\quad$ Conditions & Final results $\quad$ Conditions & Final results Conditions \\
\hline
\end{tabular}




\begin{tabular}{l|cc|cc|lc}
\hline Patient 1 & $(0.0365,0.9360)$ & 3 & $(0.9455,0.0074)$ & 3 & 0.7049 & 3 \\
Patient 2 & $(0.0310,0.9446)$ & 1 & $(0.9537,0.0045)$ & 2 & 0.8955 & 2 \\
Patient 3 & $(0.0401,0.9199)$ & 5 & $(0.8832,0.0172)$ & 4 & 0.3390 & 5 \\
Patient 4 & $(0.0333,0.9445)$ & 2 & $(0.9652,0.0026)$ & 1 & 0.9678 & 1 \\
Patient 5 & $(0.0420,0.9349)$ & 4 & $(0.8809,0.0383)$ & 5 & 0.4350 & 4 \\
\hline
\end{tabular}

From the above table, we can see that the determination of classifying patients is diversified with the three decision making methods, in which the proposed method, the intuitionistic fuzzy decision making method with WAO and the intuitionistic fuzzy TOPSIS indicate Patient 3 and Patient 5 should be treated in West China Hospital. . Furthermore, the decision rankings derived by the latter two methods are closer to each other, whereas the decision ranking derived by the proposed method is more different with the other two rankings.

The thermodynamic method for MCDM with IFNs, which considers both the quantity and quality of the decision information, obtains a ranking of the patients, which is majorly different from the rankings obtained by the method with WAO and the TOPSIS for MCDM in intuitionistic fuzzy environment. Meanwhile, the other two decision making methods, which only make decisions according to the quantity of the data, present the two more similiar rankings of the patients. In most situations, the DMs' opinions are not the same, or even much different from each other. If we only consider the values of the data provided by the DMs but not the distributions of the values, then we will overlook a significant character of the data and lose some very useful decision information. From this point of view, the proposed method provides a new angle to the available data and is 
much more comprehensive and reliable.

With the complexity of the decision making environment and limitations of human thinking, the DMs are hard to obtain or provide much decision making information to objects. Since the quality of the decision making information is a crucial and influent factor to the decision making results, it is necessary to be considered in the general decision making problems to fully capture the potential significance of known information. Therefore, the proposed method carries important implications for the application problems with many uncertainties and complexities, such as medical decision support, medical risk management, emergency decision making, etc.

\section{Conclusions}

The knowledge structure of thermodynamics provides us a useful tool to depict the stability (or instability) of a system, while the IFNs can comprehensively describe the uncertainty of objects. In this paper, we propose the thermodynamic method for MCDM with IFNs to the problems in intuitionistic fuzzy environment, which is highly effective by taking the quantity and quality of the decision information into consideration in the decision making process. The simulations are conducted to compare the results obtained by the proposed method and other two commonly used methods, and show that the quality of the decision information can strongly impact the ranking of the alternatives. Furthermore, we apply the proposed thermodynamic method for MCDM with IFNs to assist the hierarchical medical system in West China Hospital to show the comprehensiveness and dependability of the proposed method.

With the increasing complexity of the decision making problems in real life, the uncertainty of 
objects is necessary to be considered, and the more information of the decision data should be mined to let the decision making process be more reasonable and more reliable. Toward to this goal, the thermodynamic method for MCDM with IFNs has been proposed in the paper. On the one hand, the proposed method can portray the fuzziness of objects well. On the other hand, it can simultaneously consider the quantity and quality of the decision information in the decision making process.

Until now, this paper mainly focuses on the MCDM problems with decision matrixes, and thus, in our further research, we will make more efforts to utilize the knowledge structure of thermodynamics to the decision making process with preference relations.

\section{Acknowledgements}

The work was supported by the National Natural Science Foundation of China (No. 71571123, No. 71501135, No. 61273209), the Program for Changjiang Scholars at Sichuan University (No. YJ201430) and the Young scholars high level academic team construction project at Sichuan University (skgt201501).

\section{References}

[1] M.M. Wiecek, M. Ehrgott, G. Fadel, J.R. Figueira, Multiple criteria decision making for engineering, Omega, vol. 36, pp: 337-339, 2008.

[2] Y.H. Hung, S.C.T. Chou, G.H. Tzeng, Knowledge management adoption and assessment for SMEs by a novel MCDM approach, Decision Support Systems, vol. 51, pp: 270-291, 2011.

[3] T. Gürbüz, Y.E. Albayrak, An engineering approach to human resources performance 
evaluation: Hybrid MCDM application with interactions, Applied Soft Computing, vol. 21, pp: 365-375, 2014.

[4] S. Ghosh, T. Chakraborty, S. Saha, M. Majumder, M. Pal, Development of the location suitability index for wave energy production by ANN and MCDM techniques, Renewable and Sustainable Energy Reviews, vol. 59, pp: 1017-1028, 2016.

[5] J.R. Chang, C.H. Cheng, L.S. Chen, A fuzzy-based military officer performance appraisal system, Applied Soft Computing, vol. 7, pp: 936-945, 2007.

[6] K.P. Lin, K.C. Hung, An efficient fuzzy weighted average algorithm for the military UAV selecting under group decision-making, Knowledge-Based Systems, vol. 24, pp: 877-889, 2011.

[7] J.M. Sánchez-Lozano, J. Serna, A. Dolón-Payán, Evaluating military training aircrafts through the combination of multi-criteria decision making processes with fuzzy logic. A case study in the Spanish Air Force Academy, Aerospace Science and Technology, vol. 42, pp: 58-65, 2015.

[8] H.K. Chiou, G.H. Tzeng, D.C. Cheng, Evaluating sustainable fishing development strategies using fuzzy MCDM approach, Omega, vol. 33, pp: 223-234, 2005.

[9] Y.H. Hung, T.L. Huang, J.C. Hsieh, H.J. Tsuei, C.C. Cheng, G.H. Tzeng, Online reputation management for improving marketing by using a hybrid MCDM model, Knowledge-Based Systems, vol. 35, pp: 87-93, 2012.

[10] A. Bonetti, S. Bortot, M. Fedrizzi, R.A. Marques Pereira, A. Molinari, Modelling group processes and effort estimation in project management using the Choquet integral: An MCDM approach, Expert Systems with Applications, vol. 39, pp: 13366-13375, 2012.

[11] E. Mulliner, N. Malys, V. Maliene, Comparative analysis of MCDM methods for the assessment of sustainable housing afford ability, Omega, vol. 59, pp: 146-156, 2016.

[12] C.L Hwang, K.S Yoon, Multiple attribute decision methods and applications, Berlin, Germany: Springer, 1981.

[13] B. Roy, P. Bertier, La méthode ELECTRE II: une méthode de classement en prédence de critères multiples, 1971.

[14] S. Opricovic, Multi-criteria optimization of civil engineering systems, Faculty of Civil Engineering, Belgrade, 1998.

[15] L. Gomes, M. Lima, TODIM: Basic and application to multi-criteria ranking of projects with 
environmental impacts, Foundations of Computing and Decision Sciences, vol. 16, pp: 113-127, 1992.

[16] E.K. Zavadskas, A. Kaklauskas, V. Sarka, The new method of multi-criteria complex proportional assessment of projects, Technological and Economic Development of Economy, vol. 3, pp: 131-139,1994.

[17] https://en.wikipedia.org/wiki/Weighted_arithmetic_mean.

[18] K. Charles, K. Herbert, Thermal Physics, Macmillan, ISBN: 9780716710882, 1980.

[19] P. Perrot, A to Z of Thermodynamics, Oxford University Press, ISBN: 9780198565529, 1998.

[20] https://en.wikipedia.org/wiki/Entropy.

[21] https://en.wikipedia.org/wiki/First_law_of_thermodynamics.

[22] https://en.wikipedia.org/wiki/Second_law_of_thermodynamics.

[23] https://en.wikipedia.org/wiki/Third_law_of_thermodynamics.

[24] L.A. Zadeh, Fuzzy sets, Information and Control, vol. 8, pp: 338-356, 1965.

[25] K. Atanassov, Intuitionistic fuzzy sets, Sofia. In: Sgurev Ved: VII ITKR's Session, 1983.

[26] K. Atanassov, Intuitionistic fuzzy set, Fuzzy Sets and Systems, vol. 20, PP: 87-96, 1986.

[27] F.E. Boran, S. Genç, M. Kurt, D. Akay, A multi-criteria intuitionistic fuzzy group decision making for supplier selection with TOPSIS method, Expert Systems with Applications, vol. 36, pp: 11363-11368, 2009.

[28] M.C. Wu, T.Y. Chen, The ELECTRE multicriteria analysis approach based on Atanassov's intuitionistic fuzzy sets, Expert Systems with Applications, vol. 38, pp: 12318-12327, 2011.

[29] K. Devi, Extension of VIKOR method in intuitionistic fuzzy environment for robot selection, Expert Systems with Applications, vol. 38, pp: 14163-14168, 2011.

[30] Z.S. Xu, H.C. Liao, Intuitionistic fuzzy analytic hierarchy process, IEEE Transactions on Fuzzy Systems, vol. 22, pp: 749-761, 2014

[31] E.K. Zavadskas, J. Antucheviciene, S.H.R. Hajiagha, S.S. Hashemi, Extension of weighted aggregated sum product assessment with interval-valued intuitionistic fuzzy numbers (WASPAS-IVIF), Applied Soft Computing, vol. 24, pp: 1013-1021, 2014.

[32] D.F. Li, Extension of the LINMAP for multi-attribute decision making under Atanassov's intuitionistic fuzzy environment, Fuzzy Optimization and Decision Making, vol. 7, pp: 17-34, 
2008.

[33] T.Y. Chen, An interval-valued intuitionistic fuzzy LINMAP method with inclusion comparison possibilities and hybrid averaging operations for multiple criteria group decision making, Knowledge-Based Systems, vol. 45, pp: 134-146, 2013.

[34] T.Y. Chen, The inclusion-based LINMAP method for multiple criteria decision analysis within an interval-valued Atanassov's intuitionistic fuzzy environment, International Journal of Information Technology \& Decision Making, vol. 13, pp: 1325-1360, 2014.

[35] Z.S. Xu, R.R. Yager, Some geometric aggregation operators based on intuitionistic fuzzy sets, International Journal of General Systems, vol. 35, pp: 417-433, 2006.

[36] Z.S. Xu, Intuitionistic fuzzy aggregation operations, IEEE Transactions on Fuzzy Systems, vol. 15, pp: 1179-1187, 2007.

[37] Q. Lei, Z.S. Xu, Derivative and differential operations of intuitionistic fuzzy numbers, Journal of Intelligent Systems, vol. 30, pp: 468-498, 2015.

[38] H. Bustince, P. Burillo, Correlation of interval-valued intuitionistic fuzzy sets, Fuzzy Sets and Systems, vol. 74, pp: 237-244, 1995. 